\title{
Diverging in vitro inflammatory responses toward Streptococcus uberis in mouse macrophages either preconditioned or continuously treated with $\beta$-hydroxybutyrate
}

\author{
T. H. Swartz, ${ }^{1,2 *} \odot$ B. J. Bradford, ${ }^{1,2} \odot$ and L. K. Mamedova ${ }^{1,2} \odot$
}

\section{Graphical Abstract}

Preconditioning BHB treatment

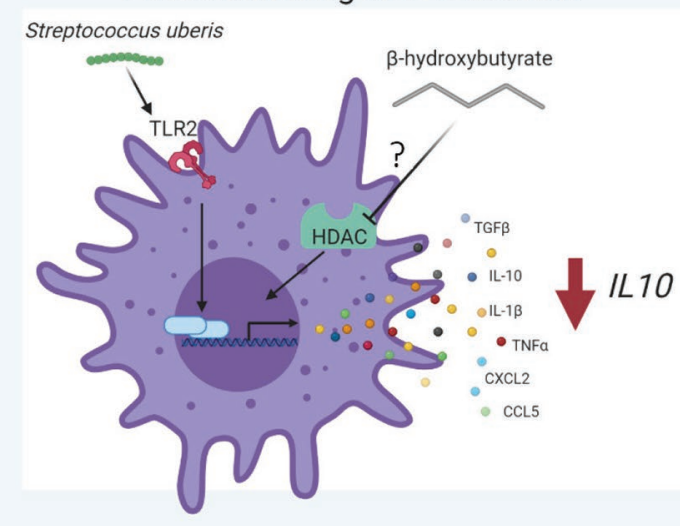

Continuous BHB treatment

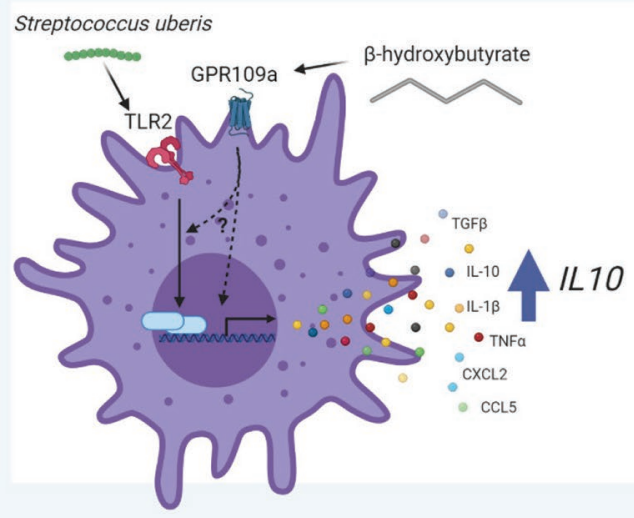

\section{Summary}

Elevated ketone concentrations have been associated with mastitis in postpartum dairy cows. Therefore, we sought to determine the effect of a ketone on macrophage inflammatory responses during a Streptococcus uberis challenge. When cells were pretreated with a ketone before $S$. uberis challenge, mRNA abundance of a membrane receptor for a bacterial cell wall component was reduced. However, when cells were treated during the challenge, the opposite was observed. These responses could help explain the association of ketosis with mastitis.

\section{Highlights}

- $\quad \beta$-Hydroxybutyrate preconditioning reduced T/r2 and tended to reduce $/ / 10$ expression.

- Continuous $\beta$-hydroxybutyrate treatment increased T/r2 and //10 expression.

- $\quad$ Diverging responses due to the timing of BHB treatment suggest opposing mechanisms.

\footnotetext{
${ }^{1}$ Department of Animal Science, Michigan State University, East Lansing $48824,{ }^{2}$ Department of Animal Sciences and Industry, Kansas State University, Manhattan 66506. *Corresponding author: swartztu@msu.edu. $\odot 2021$, The Authors. Published by Elsevier Inc. and Fass Inc. on behalf of the American Dairy Science Association ${ }^{\oplus}$. This is an open access article under the CC BY license (http://creativecommons.org/licenses/by/4.0/). Received October 08, 2020. Accepted January 10, 2021.
} 


\title{
Diverging in vitro inflammatory responses toward Streptococcus uberis in mouse macrophages either preconditioned or continuously treated with $\beta$-hydroxybutyrate
}

\author{
T. H. Swartz, ${ }^{1,2 *} \odot$ B. J. Bradford, ${ }^{1,2} \odot$ and L. K. Mamedova ${ }^{1,2} \odot$
}

\begin{abstract}
Hyperketonemia is a common condition in early-lactation dairy cows that has been associated with an increase in the risk of infectious disease. Recent mouse studies have elucidated an anti-inflammatory effect of the ketone body $\beta$-hydroxybutyrate (BHB). Therefore, the objective of this study was to determine whether BHB altered inflammatory responses in macrophages challenged with the common mastitis pathogen Streptococcus uberis. A secondary objective was to determine whether the inflammatory response to the $S$. uberis challenge was dependent on whether BHB was present in the medium during the challenge (i.e., preconditioned vs. continuous treatment). Two cell culture experiments were conducted. In the first experiment, mouse macrophages (RAW 264.7 line) were preconditioned with BHB $(0,0.6,1.2$, and $1.8 \mathrm{mM})$ for $24 \mathrm{~h}$; the medium was then replaced with a standard cell culture medium, and the cells were challenged or not with $S$. uberis for an additional $6 \mathrm{~h}$. In the second experiment, a similar protocol was used; however, cells were preconditioned with $\mathrm{BHB}(0,0.6,1.2$, and $1.8 \mathrm{mM})$ for $24 \mathrm{~h}$, the medium was replaced with fresh medium containing the same concentration of BHB, and cells were either challenged or not with $S$. uberis for $6 \mathrm{~h}$. In both experiments, relative transcript abundance of cell membrane receptors (Tlr2 and Gpr109a), cytokines (Illb, IllO, Tnf, and Tgfbl), and chemokines (Cxcl2 and Ccl5) were determined using quantitative real-time PCR and normalized against the geometric mean of Hprt and $B 2 \mathrm{~m}$. Data were analyzed using a linear mixed model, and orthogonal contrasts were conducted to examine the effect of S. uberis challenge and BHB treatment. Streptococcus uberis activated the macrophages, noted by greater transcript abundance of analyzed genes. Intriguingly, in both experiments, the $S$. uberis challenge increased expression of Gpr109a, which encodes a receptor that is ligated by BHB. Paradoxically, preconditioning macrophages with BHB increased transcript abundance of the immunosuppressive cytokine $T g f b l$ and increased that of the neutrophil chemoattractant Cxcl2. Preconditioning decreased Tlr2 and tended to decrease $I l 10$ transcript abundance. In opposition to the preconditioning experiment, continuous treatment of BHB during the $S$. uberis challenge linearly increased abundance of Tlr 2 and $I l 10$ transcripts. Continuous BHB treatment also increased expression of $I l l b$. In conclusion, BHB treatment altered macrophage inflammatory responses during an $S$. uberis challenge; however, the direction of this response was dependent on whether BHB was added to the medium during the S. uberis challenge. Future studies should be conducted using bovine macrophages and in vivo approaches to examine BHB effects during an $S$. uberis challenge.
\end{abstract}

$\mathrm{M}$ astitis is the most common and costly disease in the dairy industry. The incidence of clinical mastitis is dramatically greater during the first few weeks of lactation (van den Borne et al., 2010; Hammer et al., 2012). At the beginning of lactation, a depression of feed intake occurs simultaneously with an increase in energy demand, resulting in negative energy balance. Consequently, dairy cattle mobilize fat reserves, and some of these fatty acids are transported to the liver for ATP production. However, not all fatty acids are completely oxidized, resulting in the production of BHB, a major ketone body. Hyperketonemia is defined as an abnormal increase in circulating ketone bodies that may result in the health disorders more commonly known as clinical and subclinical ketosis. These disorders have been associated with greater incidence in early lactation dairy cattle of infectious diseases, including mastitis (Oltenacu and Ekesbo, 1994; Raboisson et al., 2014). Alarmingly, a large field study reported that $43 \%$ of post- partum dairy cows have subclinical ketosis ( $\geq 1.2 \mathrm{~m} M \mathrm{BHB}$; McArt et al., 2012), underscoring the potential implications of subclinical ketosis for infectious disease incidence.

Numerous studies have demonstrated that BHB impairs immune function (Suriyasathaporn et al., 2000). Indeed, BHB treatment reduced the ability of neutrophils to kill bacteria (Grinberg et al., 2008), impaired lymphocyte proliferation (Franklin et al., 1991) and antibody production (Nonnecke et al., 1992), and reduced phagocytosis in bovine milk macrophages (Klucinski et al., 1988a). Streptococcus uberis is a common environmental mastitis pathogen (Oliver, 1988; Jayarao et al., 1999; Riekerink et al., 2008). Coinciding with negative energy balance, environmental streptococci have a greater IMI rate during the first month of lactation compared with the remainder of the lactation (Todhunter et al., 1995), suggesting that hyperketonemia may be a risk factor for $S$. uberis infections. Curiously, S. uberis infections result in

\footnotetext{
${ }^{1}$ Department of Animal Science, Michigan State University, East Lansing $48824,{ }^{2}$ Department of Animal Sciences and Industry, Kansas State University, Manhattan 66506. *Corresponding author: swartztu@msu.edu. @ 2021, The Authors. Published by Elsevier Inc. and Fass Inc. on behalf of the American Dairy Science Association ${ }^{\circledast}$. This is an open access article under the CC BY license (http://creativecommons.org/licenses/by/4.0/). Received October 08, 2020. Accepted January 10, 2021.
} 
an uncontrolled inflammatory response due to sustained migration of neutrophils (Thomas et al., 1994); however, this influx of leukocytes is generally not well correlated with reductions in bacterial counts (colony-forming units; Pedersen et al., 2003; Rambeaud et al., 2003; Bannerman et al., 2004). Macrophages are critical innate immune cells responsible not only for killing bacterial pathogens but also for recruiting additional immune cells to the inflammatory site. Although debatable (Denis et al., 2006), past research has suggested that macrophages play a more pivotal role than neutrophils in $S$. uberis clearance (Thomas et al., 1994). Therefore, the objective of this experiment was to examine the effect of BHB on inflammatory mediators from macrophages during an S. uberis challenge. A secondary objective was to determine whether the inflammatory response to the $S$. uberis challenge was dependent on whether BHB was present in the medium during the challenge (i.e., preconditioned vs. continuous treatment). We hypothesized that BHB would attenuate inflammatory responses demonstrated by shifts in cytokine transcript abundance toward an anti-inflammatory profile.

A wild-type strain of $S$. uberis (provided by Dr. PeterssonWolfe, Department of Dairy Science, Virginia Tech, Blacksburg) was originally isolated from a dairy cow with mastitis and stored in $10 \%$ skim milk at $-80^{\circ} \mathrm{C}$. Bacteria were streaked on an esculin blood agar plate and incubated overnight. Five colonies were then cultured in Todd-Hewitt broth and incubated for $7 \mathrm{~h}$ at $37^{\circ} \mathrm{C}$ on an orbital shaker (200 rpm). The bacterial suspension was pelleted by centrifugation at $1,600 \times g$ for $20 \mathrm{~min}$, washed with sterile PBS, and resuspended in Dulbecco's modified Eagle medium (SigmaAldrich) containing 1\% L-glutamine and 10\% heat-inactivated fetal bovine serum. Serial dilutions were used to achieve the desired concentration for challenge, and the challenge inoculum concentration $\left(2.5 \times 10^{5} \mathrm{cfu}\right)$ was verified using drop plating onto esculin blood agar.

Mouse macrophages (RAW 264.7 line) were cultured in Dulbecco's modified Eagle medium supplemented with 1\% L-glutamine, $10 \%$ heat-inactivated fetal bovine serum, and $0.2 \%$ penicillinstreptomycin. Twenty-four-well plates were seeded with $1 \times 10^{5}$ cells per well and incubated in a humidified atmosphere for $24 \mathrm{~h}$ at $37^{\circ} \mathrm{C}$ and $5 \% \mathrm{CO}_{2}$. To maintain a neutral $\mathrm{pH}$ in culture medium, BHB was added as sodium salt, and a treatment group with $1.8 \mathrm{mM}$ added $\mathrm{NaCl}$ was included as an osmotic control (OC). Two experiments were conducted: (1) a BHB preconditioning experiment with no BHB added to the medium during the $S$. uberis challenge, and (2) a continuous BHB experiment with a 24-h preconditioned BHB treatment followed by BHB treatment during the $S$. uberis challenge. Experiment 1 assessed the effects of BHB preconditioning on macrophage inflammatory responses during an S. uberis challenge, whereas experiment 2 assessed the interaction of BHB treatment with $S$. uberis challenge in addition to a preconditioning effect. In experiment 1 , cells were preconditioned with BHB (Sigma-Aldrich) at various concentrations $(0,0.6,1.2$, or $1.8 \mathrm{mM})$ for $24 \mathrm{~h}$. After the 24-h incubation step, the medium was removed and fresh medium without antibiotics and with or without $2.5 \times$ $10^{5} \mathrm{cfu}$ of $S$. uberis was added for $6 \mathrm{~h}$. In experiment 2, a similar protocol was used; however, cells were preconditioned with $\mathrm{BHB}$ for $24 \mathrm{~h}$, and then the cells were challenged or not with $2.5 \times 10^{5}$ cfu $S$. uberis by replacing the medium with fresh medium without antibiotics, but still containing the same BHB concentration as used during the preconditioning for a total of $30 \mathrm{~h}$ of incubation with BHB (24 h of preconditioning plus $6 \mathrm{~h}$ during the S. uberis challenge).

To measure transcript abundance, cells ( $n=8$ wells per treatment group) were placed on an ice pack, the medium was aspirated, and the cells were washed once with cold PBS and then lysed using a 1\% 2-mercaptoethanol RLT lysis buffer (Qiagen). Cell lysates were stored at $-80^{\circ} \mathrm{C}$, and total RNA was isolated within $3 \mathrm{~d}$ of freezing using the RNeasy kit (Qiagen). RNA was quantified using spectroscopy, and purity was assessed using the 260/280 nm absorbance ratio. Complementary DNA was synthesized immediately following RNA isolation; RNA integrity was not assessed. Total RNA ( 0.9 and $1 \mu \mathrm{g}$ for experiments 1 and 2, respectively) was used as a template for the reverse transcription reaction using random primers (Bio-Rad Laboratories Inc.). Quantitative real-time PCR was performed (7500 Fast Real-Time PCR System, Applied Biosystems) in duplicate with $200 \mathrm{n} M$ gene-specific forward and reverse primers with iTaq Universal SYBR Green Supermix (BioRad Laboratories Inc.). Primers were designed from mouse GenBank sequences to amplify an intron-spanning region of the gene and validated by identifying a single amplicon from the melt curve analysis. Primer efficiencies were calculated using a 5-point curve (Table 1). Transcript abundance was quantified using the relative expression ratio from Pfaffl (2001), with the geometric mean of Hprt and $B 2 m$ used to normalize values.

To determine whether BHB treatment influenced cell viability, cellular metabolism was assessed using resazurin as a proxy for cell viability (Riss et al., 2004). In black-walled, clear-bottomed 96-well plates, $20 \mu \mathrm{L}$ of a $0.15 \mathrm{mg} / \mathrm{mL}$ solution of resazurin sodium salt (Sigma-Aldrich) was added directly to RAW 264.7 cells in $200 \mu \mathrm{L}$ of medium following BHB treatments ( $\mathrm{n} \geq 8$ wells per treatment group). These plates were then incubated for another $4 \mathrm{~h}$ to allow conversion of resazurin to resorufin. Cellular metabolism was assessed by measuring absorbance at $570 \mathrm{~nm}$ using a plate reader (Synergy HTX; BioTek Instruments Inc.) and Gen5 software (BioTek Instruments Inc.). Results are expressed as a percentage of the control.

Statistical analyses were conducted using PROC GLIMMIX (SAS 9.4, SAS Institute Inc.), and each experiment was analyzed separately. The model included the fixed effects of treatment and the random effect of cell culture plate. For cell viability, orthogonal contrasts (LSMESTIMATE statement with Bonferroni adjustment) were performed to test the overall effect of BHB (OC vs. BHB treatment groups), as well as linear and quadratic contrasts to test BHB dose responses. For transcript abundance, orthogonal contrasts were performed to test the effect of $S$. uberis (unchallenged control and OC vs. OC $+S$. uberis), overall effect of BHB within $S$. uberis-challenged treatment groups $(\mathrm{OC}+S$. uberis vs. S. uberischallenged groups with either $0.6,1.2$, or $1.8 \mathrm{~m} M$ BHB treatments), as well as linear and quadratic contrasts to test BHB dose responses. To meet the assumption of normality (PROC UNIVARIATE), all response variables required natural logarithmic transformation. An outlier was defined if the observation had a studentized residual $>3$ in absolute value, and therefore was removed from the analysis. Significance was declared at $P \leq 0.05$ and trends at $0.05<P<0.10$.

Cellular metabolism was assessed $24 \mathrm{~h}$ posttreatment as a proxy for cell viability. Treatment (overall BHB effect, $P=0.32$ ) did not influence cellular metabolism of resazurin $(\mathrm{LSM} \pm \mathrm{SE}$ expressed as 
Table 1. Sequence ( $F$, forward; $R$, reverse), accession number, and primer efficiency for analyzed transcripts

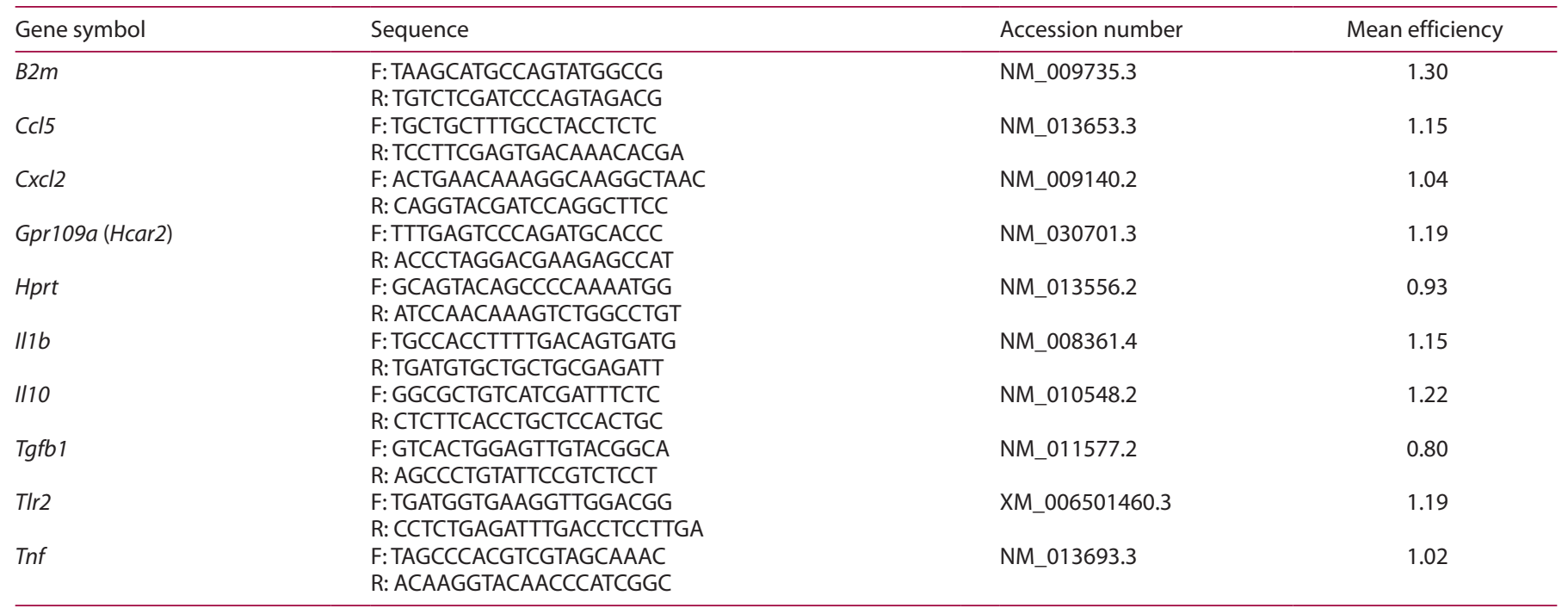

a percentage of the control; $\mathrm{CON}, 100 \pm 2 \%$; OC, $95 \pm 2 \%$; $0.6 \mathrm{mM}$ BHB, $91 \pm 3 \% ; 1.2 \mathrm{mM}$ BHB, $87 \pm 3 \%$; $1.8 \mathrm{~m} M$ BHB, $94 \pm 3 \%$ ). For mRNA abundance, we first assessed the effects of $S$. uberis and BHB treatment on the geometric mean of the cycle threshold $(\mathrm{Ct})$ values of $B 2 \mathrm{~m}$ and Hprt to validate the internal control genes. Neither $S$. uberis nor BHB influenced the control genes $(S$. uberis effect, $P=1.00$; overall BHB effect, $P=1.00)$. As expected, $S$. uberis activated the macrophages, as shown by greater transcript abundance of Tlr2, Gpr109a, Ccl5, Cxcl2, Illb, Il10, Tgfb1, and $\operatorname{Tnf}$ (all $P<0.01$ ) compared with unchallenged macrophages (Figure 1). $\beta$-Hydroxybutyrate preconditioning reduced Tlr2 (overall BHB effect, $P=0.04$ ) and tended to reduce $I l 10$ (overall BHB effect, $P=0.07$ ) transcript abundance. Conversely, BHB preconditioning increased $\mathrm{Cxcl} 2$ (overall BHB effect, $P=0.02$ ) and increased $T g f b 1$ in a dose-dependent manner (overall BHB effect, $P<0.01$; linear BHB effect, $P=0.02$ ).

Cellular metabolism was assessed $30 \mathrm{~h}$ following BHB treatment as a proxy for cell viability. Treatment (overall BHB effect, $P=1.00$ ) did not influence cellular metabolism of resazurin (LSM $\pm \mathrm{SE}$; $\mathrm{CON}, 100 \pm 3 \%$; OC, $91 \pm 5 \%$; $0.6 \mathrm{mM}$ BHB, $93 \pm 3 \%$; 1.2 $\mathrm{m} M \mathrm{BHB}, 96 \pm 3 \% ; 1.8 \mathrm{~m} M \mathrm{BHB}, 95 \pm 3 \%$ ). Furthermore, neither S. uberis nor BHB influenced the geometric mean of the $\mathrm{Ct}$ values of the internal control genes $B 2 m$ and Hprt $(S$. uberis effect, $P=$ 0.63 ; overall BHB effect, $P=1.00$ ). As in the previous experiment, $S$. uberis activated the macrophages, noted by greater transcript abundance of Tlr2, Gpr109a, Ccl5, Cxcl2, Il1b, Il10, Tgfb1, and $\operatorname{Tnf}$ (all $P<0.01$ ) compared with unchallenged macrophages (Figure 2). In opposition to the preconditioning experiment, continuous BHB treatment increased Tlr2 (overall BHB effect, $P=0.03$; linear $\mathrm{BHB}, P=0.04$ ) and $1 l 10$ (overall BHB effect, $P=0.05$; linear $\mathrm{BHB}, P=0.02)$ transcript abundance in a linear manner. Similarly, BHB treatment dose-dependently increased $1 l 1 b$ abundance (overall BHB effect, $P<0.01$; linear BHB, $P<0.01$ ).

Numerous studies have examined the effect of BHB on bovine immune function using in vitro methods (Klucinski et al., 1988a,b; Franklin et al., 1991; Nonnecke et al., 1992; Suriyasathaporn et al., 1999; Grinberg et al., 2008; Ster et al., 2012; Carretta et al., 2020).
The general consensus of these studies is that BHB impairs functionality of immune cells, through reduced phagocytosis, antibody production, proliferation, and dysregulated chemotaxis, depending on the immune cell discussed. Our study is the first to show that BHB altered inflammatory responses in macrophages challenged with $S$. uberis and that these effects are dependent on the presence of the $\mathrm{BHB}$ in the medium during the challenge.

In our study, BHB preconditioning reduced Tlr 2 and tended to reduce $I l 10$ in a dose-dependent manner, although no effect was found on the proinflammatory cytokines $\operatorname{Tn} f$ and $I l 1 b$. Similar results were reported with butyrate preconditioning of macrophages, which enhanced antimicrobial functions toward Salmonella, reduced the anti-inflammatory cytokine IL-10, and had no effect on TNF- $\alpha$ or IL- $1 \beta$ protein or transcript abundance (Schulthess et al., 2019). The likely mechanism behind the BHB preconditioning effect is inhibition of histone deacetylase (Shimazu et al., 2013; Newman and Verdin, 2014). As the name suggests, histone deacetylases are enzymes that remove acetyl groups from histones (Gregoretti et al., 2004) and, by doing so, these enzymes regulate gene expression.

$\beta$-Hydroxybutyrate preconditioning increased $\mathrm{Tg} f b 1$ and $\mathrm{Cxcl} 2$ during an $S$. uberis challenge. Transforming growth factor- $\beta$ is a pleiotropic cytokine involved in many cellular functions, including proliferation, differentiation, and regeneration, as well as suppressing inflammatory responses (Weiss and Attisano, 2013), whereas CXCL2 is a chemoattractant for neutrophils produced by macrophages in response to $S$. uberis (Günther et al., 2016). To the best of our knowledge, this is the first study to report BHB preconditioning effects on $T g f b 1$ and $C x c l 2$. Considering the various alterations in cytokine transcript abundance noted in this experiment, it is difficult to reconcile the results because many of these differentially expressed cytokines have opposing effects. As such, in vivo studies are needed to better illustrate whether these BHB effects can alter mammary gland defenses during an $S$. uberis infection.

Continuous BHB treatment dose-dependently increased Tlr2, $I l 1 b$, and $I l 10$. Toll-like receptor 2 is a critical cell membrane receptor for identification and elimination of S. uberis (Li et al., 

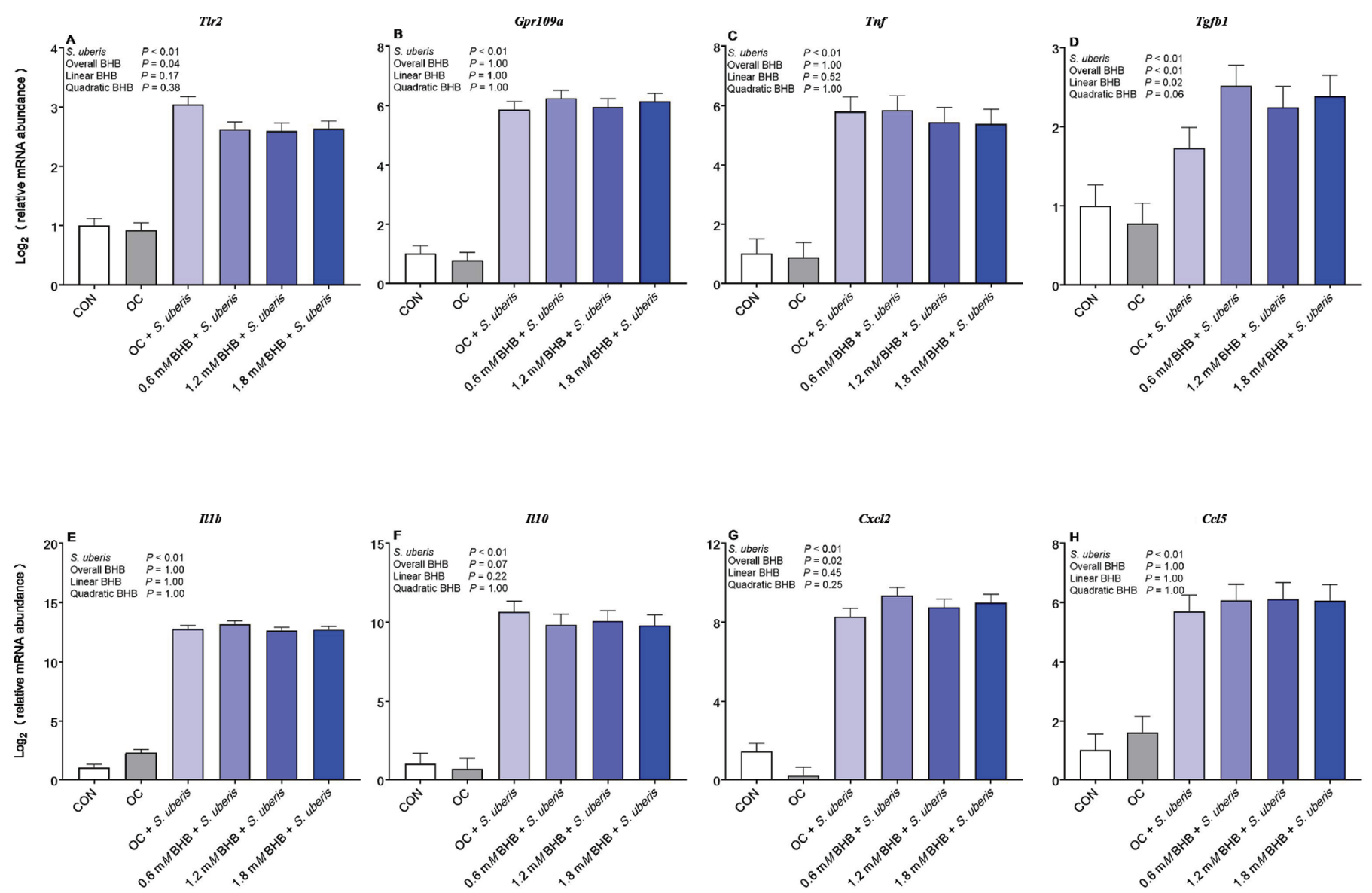

Figure 1. Effect of BHB preconditioning during a Streptococcus uberis challenge on transcript abundance of genes involved in inflammation. $\beta$-Hydroxybutyrate preconditioning reduced $\operatorname{TI} 2(\mathrm{~A})$ and tended to reduce $/ 110(\mathrm{~F})$, while increasing $\operatorname{Tg} f b 1(\mathrm{D})$ and $C x \mathrm{C} / 2(\mathrm{G})$ transcript abundance during an $\mathrm{S}$. uberis challenge. Treatment groups ( $n=8$ replicates/treatment group) include control (CON), osmotic control (OC; $1.8 \mathrm{mM} \mathrm{NaCl),} 0.6 \mathrm{mM} \mathrm{BHB}, 1.2 \mathrm{mM} \mathrm{BHB}$, and $1.8 \mathrm{mM} \mathrm{BHB}$. Treatments were applied $24 \mathrm{~h}$ before challenge; the medium was then removed and replaced with fresh medium containing $2.5 \times 10^{5} \mathrm{cfu}$ of $S$. uberis for $6 \mathrm{~h}$. $\beta$-Hydroxybutyrate was not added to the medium during the 6 -h challenge.

2020). Although it is not clear why BHB treatment increased Tlr2, we speculate that this could be a response to impaired phagocytosis, as has been previously shown with ketone bodies (Klucinski et al., 1988a). Ultimately, a reduction in phagocytosis would result in greater $S$. uberis counts in the cell culture medium, leading to greater activation of TLR2, which would in turn drive greater transcription of proinflammatory genes including $T l r 2$ and $I l l b$. An increase in the anti-inflammatory cytokine $I l 10$ is consistent with past studies examining BHB (Zarrin et al., 2014; Chen et al., 2018) or butyrate effects (Singh et al., 2014) during an inflammatory insult. Streptococcus uberis increased Gpr109a, which is a receptor for BHB that has known anti-inflammatory effects when activated (Blad et al., 2012) by augmenting IL-10 production (Al-odaini and Singh, 2019). This is intriguing, because these data could imply that $S$. uberis impairs the immune system's ability to kill this pathogen by increasing GPR109A expression on macrophages and subsequently driving an IL-10 response.

We found diverging effects on Tlr2 and Il10 between preconditioned and continuous BHB treatment of macrophages. When reading the literature evaluating treatment effects on cellular functions, it is easy to reach quick conclusions with little regard to when treatments were applied. Here, we provide a cautionary finding because the effects of BHB on inflammation-related transcripts were dependent on whether the BHB was present in the medium during the challenge. In light of these results, our data promote some reconsideration of preconditioning effects, especially because this is a popular treatment protocol used in cell culture studies. Moreover, although we find the preconditioning effects intriguing, it seems more likely that a continuous treatment would more closely reflect what occurs in vivo.

In conclusion, S. uberis is responsible for a large proportion of mastitis during the first month of lactation, suggesting that hyperketonemia may be a risk factor for this pathogen. Preconditioning macrophages with BHB resulted in a decrease in Tlr2 and tended to decrease $I l 10$, yet in continuously treated cells, BHB treatment increased the abundance of these transcripts. Altered cytokine transcript abundance could be indicative of the immune dysfunction that is typically seen in periparturient dairy cows. Future studies should be conducted using bovine macrophages to examine BHB effects on inflammation during a challenge, as the use of a mouse macrophage cell line may limit extrapolation to bovine mastitis. Finally, additional studies should be conducted to assess not only 

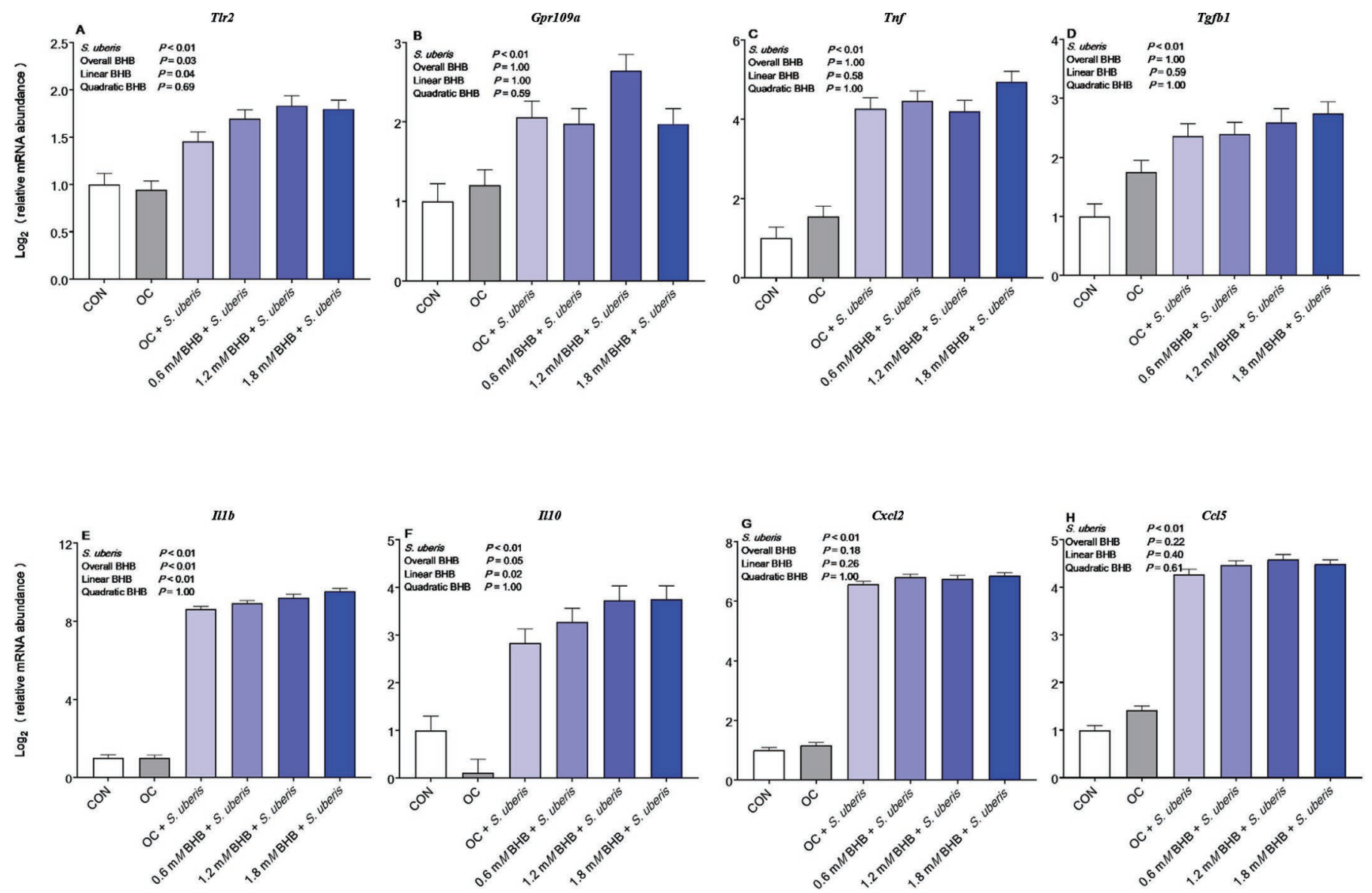

Figure 2. Effect of continuous BHB treatment during a Streptococcus uberis challenge on transcript abundance of genes involved in inflammation. $\beta$-Hydroxybutyrate treatment dose-dependently increased T/r2 (A), I/1b (E), and I/10 (F) transcript abundance during an S. uberis challenge. Treatment groups include control (CON), OC (osmotic control; $1.8 \mathrm{mM} \mathrm{NaCl}$ ), $0.6 \mathrm{mM} \mathrm{BHB}, 1.2 \mathrm{mM} \mathrm{BHB}$, and $1.8 \mathrm{mM}$ BHB. Treatments $(\mathrm{n}=8$ replicates/treatment group) were applied $24 \mathrm{~h}$ before challenge; the medium was then removed and replaced with fresh medium containing $2.5 \times 10^{5} \mathrm{cfu}$ of $\mathrm{S}$. uberis for $6 \mathrm{~h}$ but still containing the same BHB concentration as used during the preconditioning ( $24 \mathrm{~h}$ of BHB preconditioning plus 6 additional hours of BHB treatment during S. uberis challenge).

the effects of elevated concentrations of $\mathrm{BHB}$, but also the various other metabolic changes that occur in the peripartum period.

\section{References}

Al-Odaini, A., and N. Singh. 2019. Induction of IL-10 in bone-marrow derived macrophages by Helicobacter hepaticus supernatant is mediated by Gpr109a. FASEB J. 33:1b6.

Bannerman, D. D., M. J. Paape, J. P. Goff, K. Kimura, J. D. Lippolis, and J. C. Hope. 2004. Innate immune response to intramammary infection with Serratia marcescens and Streptococcus uberis. Vet. Res. 35:681-700. https:// doi.org/10.1051/vetres:2004040.

Blad, C. C., C. Tang, and S. Offermanns. 2012. G protein-coupled receptors for energy metabolites as new therapeutic targets. Nat. Rev. Drug Discov. 11:603-619. https://doi.org/10.1038/nrd3777.

Carretta, M. D., Y. Barría, K. Borquez, B. Urra, A. Rivera, P. Alarcón, M. A Hidalgo, and R. A. Burgos. 2020. $\beta$-hydroxybutyrate and hydroxycarboxylic acid receptor 2 agonists activate the AKT, ERK and AMPK pathways, which are involved in bovine neutrophil chemotaxis. Sci. Rep. 10:12491. https://doi.org/10.1038/s41598-020-69500-2.

Chen, Y., X. Ouyang, R. Hoque, I. Garcia-Martinez, M. N. Yousaf, S. Tonack, S. Offermanns, L. Dubuquoy, A. Louvet, P. Mathurin, V. Massey, B. Schnabl, R. A. Bataller, and W. Z. Mehal. 2018. $\beta$-Hydroxybutyrate protects from alcohol-induced liver injury via a Hcar2-cAMP dependent pathway. J. Hepatol. 69:687-696. https://doi.org/10.1016/j.jhep.2018.04.004.

Denis, M., N. A. Parlane, S. J. Lacy-Hulbert, E. L. Summers, B. M. Buddle, and D. N. Wedlock. 2006. Bactericidal activity of macrophages against Streptococcus uberis is different in mammary gland secretions of lactating and drying off cows. Vet. Immunol. Immunopathol. 114:111-120. https:// doi.org/10.1016/j.vetimm.2006.08.001.

Franklin, S. T., J. W. Young, and B. J. Nonnecke. 1991. Effects of ketones, acetate, butyrate, and glucose on bovine lymphocyte proliferation. J. Dairy Sci. 74:2507-2514. https://doi.org/10.3168/jds.S0022-0302(91)78428-2.

Gregoretti, I. V., Y.-M. Lee, and H. V. Goodson. 2004. Molecular evolution of the histone deacetylase family: Functional implications of phylogenetic analysis. J. Mol. Biol. 338:17-31. https://doi.org/10.1016/j.jmb.2004.02 .006 .

Grinberg, N., S. Elazar, I. Rosenshine, and N. Y. Shpigel. 2008. Beta-hydroxybutyrate abrogates formation of bovine neutrophil extracellular traps and bactericidal activity against mammary pathogenic Escherichia coli. Infect. Immun. 76:2802-2807. https://doi.org/10.1128/IAI.00051-08.

Günther, J., A. Czabanska, I. Bauer, J. A. Leigh, O. Holst, and H.-M. Seyfert. 2016. Streptococcus uberis strains isolated from the bovine mammary gland evade immune recognition by mammary epithelial cells, but not of macrophages. Vet. Res. 47:13. https://doi.org/10.1186/s13567-015-0287-8.

Hammer, J. F., J. M. Morton, and K. L. Kerrisk. 2012. Quarter-milking-, quarter-, udder- and lactation-level risk factors and indicators for clinical mas- 
titis during lactation in pasture-fed dairy cows managed in an automatic milking system. Aust. Vet. J. 90:167-174. https://doi.org/10.1111/j.1751 -0813.2012.00917.x.

Jayarao, B. M., B. E. Gillespie, M. J. Lewis, H. H. Dowlen, and S. P. Oliver. 1999. Epidemiology of Streptococcus uberis intramammary infections in a dairy herd. J. Vet. Med. B 46:433-442. https://doi.org/10.1046/j.1439 $-0450.1999 .00254 . x$.

Kluciński, W., A. Degórski, E. Miernik-Degórska, S. Targowski, and A. Winnicka. 1988a. Effect of ketone bodies on the phagocytic activity of bovine milk macrophages and polymorphonuclear leukocytes. J. Vet. Med. Ser. A 35:632-639.

Kluciński, W., E. Miernik-Degórska, A. Degórski, S. Targowski, and A. Winnicka. 1988b. Effect of ketone bodies on the mitogenic response of bovine milk lymphocytes. J. Vet. Med. Ser. A 35:626-631.

Li, B., Z. Wan, Z. Wang, J. Zuo, Y. Xu, X. Han, V. Phouthapane, and J. Miao. 2020. TLR2 signaling pathway combats Streptococcus uberis infection by inducing mitochondrial reactive oxygen species production. Cells 9:494. https://doi.org/10.3390/cells9020494.

McArt, J. A. A., D. V. Nydam, and G. R. Oetzel. 2012. Epidemiology of subclinical ketosis in early lactation dairy cattle. J. Dairy Sci. 95:5056-5066. https://doi.org/10.3168/jds.2012-5443.

Newman, J. C., and E. Verdin. 2014. Ketone bodies as signaling metabolites. Trends Endocrinol. Metab. 25:42-52. https://doi.org/10.1016/j.tem.2013 .09 .002 .

Nonnecke, B. J., S. T. Franklin, and J. W. Young. 1992. Effects of ketones, acetate, and glucose on in vitro immunoglobulin secretion by bovine lymphocytes. J. Dairy Sci. 75:982-990. https://doi.org/10.3168/jds.S0022 -0302(92)77840-0.

Olde Riekerink, R. G. M., H. W. Barkema, D. F. Kelton, and D. T. Scholl. 2008. Incidence rate of clinical mastitis on Canadian dairy farms. J. Dairy Sci. 91:1366-1377. https://doi.org/10.3168/jds.2007-0757.

Oliver, S. P. 1988. Frequency of isolation of environmental mastitis-causing pathogens and incidence of new intramammary infection during the nonlactating period. Am. J. Vet. Res. 49:1789-1793.

Oltenacu, P. A., and I. Ekesbo. 1994. Epidemiological study of clinical mastitis in dairy cattle. Vet. Res. 25:208-212.

Pedersen, L. H., B. Aalbæk, C. M. Røntved, K. L. Ingvartsen, N. S. Sorensen, P. M. H. Heegaard, and H. E. Jensen. 2003. Early pathogenesis and inflammatory response in experimental bovine mastitis due to Streptococcus uberis. J. Comp. Pathol. 128:156-164. https://doi.org/10.1053/jcpa.2002 .0620 .

Pfaffl, M. W. 2001. A new mathematical model for relative quantification in real-time RT-PCR. Nucleic Acids Res. 29:e45. https://doi.org/10.1093/ nar/29.9.e45.

Raboisson, D., M. Mounie, and E. Maigne. 2014. Diseases, reproductive performance, and changes in milk production associated with subclinical ketosis in dairy cows: A meta-analysis and review. J. Dairy Sci. 97:7547-7563. https://doi.org/10.3168/jds.2014-8237.

Rambeaud, M., R. A. Almeida, G. M. Pighetti, and S. P. Oliver. 2003. Dynamics of leukocytes and cytokines during experimentally induced Streptococcus uberis mastitis. Vet. Immunol. Immunopathol. 96:193-205. https://doi.org/ 10.1016/j.vetimm.2003.08.008

Riss, T. L., R. A. Moravec, A. L. Niles, S. Duellman, H. A. Benink, T. J. Worzella, and L. Minor. 2004. Cell viability assays. Pages 7-11 in Assay Guidance Manual. Eli Lilly \& Company and the National Center for Advancing Translational Sciences.

Schulthess, J., S. Pandey, M. Capitani, K. C. Rue-Albrecht, I. Arnold, F. Franchini, A. Chomka, N. E. Ilott, D. G. W. Johnston, E. Pires, J. McCul- lagh, S. N. Sansom, C. V. Arancibia-Cárcamo, H. H. Uhlig, and F. Powrie. 2019. The short chain fatty acid butyrate imprints an antimicrobial program in macrophages. Immunity 50:432-445.E7. https://doi.org/10.1016/ j.immuni.2018.12.018.

Shimazu, T., M. D. Hirschey, J. Newman, W. He, K. Shirakawa, N. Le Moan, C. A. Grueter, H. Lim, L. R. Saunders, R. D. Stevens, C. B. Newgard, R. V. Farese, R. de Cabo, S. Ulrich, K. Akassoglou, and E. Verdin. 2013. Suppression of oxidative stress by $\beta$-hydroxybutyrate, an endogenous histone deacetylase inhibitor. Science 339:211-214. https://doi.org/10.1126/ science. 1227166

Singh, N., A. Gurav, S. Sivaprakasam, E. Brady, R. Padia, H. Shi, M. Thangaraju, and P. D. Prasad. 2014. Activation of Gpr109a, receptor for niacin and the commensal metabolite butyrate, suppresses colonic inflammation and carcinogenesis. Immunity 40:128-139. https://doi.org/10.1016/j.immuni .2013.12.007.

Ster, C., M. C. Loiselle, and P. Lacasse. 2012. Effect of postcalving serum nonesterified fatty acids concentration on the functionality of bovine immune cells. J. Dairy Sci. 95:708-717. https://doi.org/10.3168/jds.2011-4695.

Suriyasathaporn, W., A. J. Daemen, E. N. Noordhuizen-Stassen, S. J. Dieleman, M. Nielen, and Y. H. Schukken. 1999. Beta-hydroxybutyrate levels in peripheral blood and ketone bodies supplemented in culture media affect the in vitro chemotaxis of bovine leukocytes. Vet. Immunol. Immunopathol. 68:177-186. https://doi.org/10.1016/S0165-2427(99)00017-3.

Suriyasathaporn, W., C. Heuer, E. N. Noordhuizen-Stassen, and Y. H. Schukken. 2000. Hyperketonemia and the impairment of udder defense: A review. Vet. Res. 31:397-412. https://doi.org/10.1051/vetres:2000128.

Thomas, L. H., W. Haider, A. W. Hill, and R. S. Cook. 1994. Pathologic findings of experimentally induced Streptococcus uberis infection in the mammary gland of cows. Am. J. Vet. Res. 55:1723-1728.

Todhunter, D. A., K. L. Smith, and J. S. Hogan. 1995. Environmental streptococcal intramammary infections of the bovine mammary gland. J. Dairy Sci. 78:2366-2374. https://doi.org/10.3168/jds.S0022-0302(95)76864-3.

van den Borne, B. H., G. van Schaik, T. J. Lam, and M. Nielen. 2010. Variation in herd level mastitis indicators between primi- and multiparae in Dutch dairy herds. Prev. Vet. Med. 96:49-55. https://doi.org/10.1016/j.prevetmed 2010.05.010

Weiss, A., and L. Attisano. 2013. The TGFbeta superfamily signaling pathway. WIREs Dev. Biol. 2:47-63. https://doi.org/10.1002/wdev.86.

Zarrin, M., O. Wellnitz, H. A. van Dorland, and R. M. Bruckmaier. 2014. Induced hyperketonemia affects the mammary immune response during lipopolysaccharide challenge in dairy cows. J. Dairy Sci. 97:330-339. https://doi.org/10.3168/jds.2013-7222.

\section{Notes}

T. H. Swartz @ https://orcid.org/0000-0002-9457-2418

B. J. Bradford $\odot$ https://orcid.org/0000-0002-6775-4961

L. K. Mamedova $\odot$ https://orcid.org/0000-0003-3612-0381

This work and the primary author are financially supported by a USDA postdoctoral fellowship (2019-67012-29665).

Chadron Koehn (Department of Animal Sciences and Industry, Kansas State University, Manhattan) is gratefully acknowledged for his many hours of pipetting PCR plates. The graphical abstract was created using BioRender (biorender.com).

The authors have not stated any conflicts of interest. 